\title{
A new trend in operative technique for intracerebral hemorrhage: a comparative study of stereotactic endoscopic removal and stereotactic catheter drainage
}

\author{
Myung-Hyun Kim, M.D., Ph.D., Jun-Hyeok Song, M.D., Ph.D., Sung-Hak Kim, M.D., Ph.D., \\ Dong-Bin Park, M.D., Ph.D., and Kyu-Man Shin, M.D., Ph.D.
}

This study explores the efficacy of two minimally invasive methods for evacuation of spontaneous intracerebral hemorrhage in the basal ganglia of 18 patients, some treated with stereotaxic endoscopic methods and some with a stereotaxically placed catheter within the clot for urokinase infusion and drainage. Endoscopy achieved partial removal (average 25\%), despite prolonged drug infusion and drainage. The interesting feature here is the relative inefficiency of both methods (especially fibrinolysis), although clot removal in no case was intended to remove all the hematoma. Outcomes and other patient details cannot be evaluated given the small number of cases and the lack of a randomized treatment protocol.

Click here to return to Article 2. 